\title{
Performance Evaluation of MIPv6 and HMIPv6 in terms of Key Parameters
}

\author{
Virender Kumar \\ Assistant Professor, \\ ECE Deptt,HCTM Technical \\ Campus, \\ Kaithal,Haryana-136027
}

\author{
G.C.Lall, Ph.D \\ Professor \& Chairman \\ ECE Deptt,HCTM Technical \\ Campus, \\ Kaithal, Haryana-136027
}

\author{
Pawan Dahiya, Ph.D \\ Assistant Professor \\ ECE Deptt., D.C.R.U.S.T, \\ Murthal, Haryana-131039.
}

\begin{abstract}
Mobile IPv6 (MIPv6) and Hierarchical Mobile IPv6 (HMIPv6) both are the mobility management solution proposed by the Internet Engineering Task Force (IETF) to support IP Mobility. There are various types of parameters which have been proposed and used to describe the system performance in the form of mobility of MIPv6 and HMIPv6. In this paper an analytical model have been proposed which shows the performance and applicability of MIPv6 and HMIPv6 against some key parameters in terms of cost. Numerical results demonstrate the performance of MIPv6 and HMIPv6 when certain parameters are changed.
\end{abstract}

\section{Keywords}

Mobile IPv6, Hierarchical Mobile IPv6, Access Router, Regional Size, Mobility Anchor Point.

\section{INTRODUCTION}

With the fast increasing demand for the seamless mobility providers motivate to support seamless connectivity to Mobile Nodes (MNs). To complete this aim, Internet Engineering Task Force (IETF) proposed Mobile IP (MIP) protocol. MIPv4 and MIPv6 both are the mobility management solution to maintain the on-going communication when one $\mathrm{MN}$ moves from one subnet to another. MIPv6 become the next generation solution due to the several advantages of MIPv6 over MIPv4.

\section{BACKGROUND}

\subsection{Mobile IPv6 (MIPv6)}

In Mobile IPv6 one MN is identified by two addresses: Home address and Care of address (CoA) [1]. Home Address represents the permanent address of $\mathrm{MN}$ and Care of Address (CoA) is the Temporary Address, representing the current location of MN. There is a mobility management entity i.e. Home Agent (HA) which stores the binding information of the MN. Home Agent also receives all the packets on behalf of the MN when the Correspondent Nodes do not know the current location of the MN. In MIPv6 there is a process known as "Home Registration" in which updated location is registered in HA when the MN roams in the visited networks. But in MIPv6 a frequent handover by $\mathrm{MN}$ in a local region leads to a longer signaling delay. In Handover process this longer signaling delay is the main problem of the MIPv6.

\subsection{Hierarchical Mobile IPv6 (HMIPv6)}

To solve this problem, Hierarchical Mobile IPv6 (HMIPv6) is introduced. In HMIPv6 [2] a new entity is introduced known as Mobility Anchor Point (MAP) to act as a local Home

Agent with in a region. In HMIPv6 Mobility Anchor Point (MAP) have a number of Access Routers (ARs). The number of ARs under a MAP is known as the Regional Size. In HMIPv6, there are two addresses: Regional care of address (RCoA) representing the MN's MAP \& the on-Link CoA (LCoA) representing the $\mathrm{AR}$ that the $\mathrm{MN}$ attaches to There are two types of mobility in HMIPv6: micro-mobility (handover with in a region) and macro-mobility (handover across the regions). In macro-mobility, the MN gets two new addresses: RCoA, LCoA and it will initiate a regional registration process to bind these two addresses. After having successful regional registration, the $\mathrm{MN}$ gives its new update of having new RCoA to it's HA i.e. there is a binding between its Home Address and RCoA to the HA by a Home Registration. In micro-mobility there is only a regional registration because there is no new $\mathrm{RCoA}$ of a $\mathrm{MN}$ within a region. Now, we see that in HMIPv6 when MN roams from one region to another, there is a double registration: regional registration and home registration. So in HMIPv6 the handover latency is smaller than that of MIPv6 when the MNs roam within the region but the handover latency is larger than that of MIPv6 when the MNs roam inter- region. Besides, in double registration there is a MAP processing delay leading to a longer packet delivery time because all the packets destined to MN are tunneled through MAP. So, Double registration leads to a larger handover latency and longer packet delivery time. So it is an interesting issue to select to find out the performance of MIPv6 and HMIPv6 depending upon certain conditions.

\section{PERFORMANCE ANALYSES OF MIPV6 AND HMIPV6}

\subsection{Relative Registration Cost [3]}

Definition 3.1 (Relative Registration Cost)

Relative registration cost $\left(T_{R}\right)$ is defined as the average registration time saved by using HMIPv6 compared with MIPv6 [3]

$T_{R}$ may be positive or negative. $T_{R}>0$ means the average registration delay of MIPv6 is shorter than that of HMIPv6, otherwise longer.

\section{Main Symbols in Registration Performance Analyses Symbols $\mathrm{T}_{\mathrm{R}}$ $\mathrm{T}_{\mathrm{AM}}$ \\ Definitions \\ Average registration delay of MIPv6 Average delay of delivering registration signaling over wireless link between AR and MN}




$\begin{array}{ll}\mathrm{T}_{\mathrm{HA}} & \begin{array}{l}\text { Average delay of delivering registration } \\ \text { signaling between HA and AR }\end{array} \\ \mathrm{T}_{\mathrm{H}} & \begin{array}{l}\text { Average registration signal processing } \\ \text { latency of HA }\end{array} \\ \mathrm{T}_{\mathrm{intra}} & \begin{array}{l}\text { Average delay of a registration process in } \\ \text { HMIPv6 during an intra-MAP handover } \\ \text { Average delay of a registration process in }\end{array} \\ \mathrm{T}_{\mathrm{inter}} & \begin{array}{l}\text { HMIPv6 during an inter-MAP handover } \\ \text { Average delay of delivering registration } \\ \text { signaling between MAP and AR }\end{array} \\ \mathrm{T}_{\mathrm{MA}} & \begin{array}{l}\text { Average distance between MAP and its } \\ \text { reachable ARs } \\ \mathrm{l}_{\mathrm{MA}}\end{array} \\ \mathrm{l}_{\mathrm{HA}} & \begin{array}{l}\text { Average distance between HA and AR } \\ \text { Average dwell time that an MN stays in an } \\ \mathrm{T}\end{array} \\ \mathrm{AR} & \text { Unit distance signaling transmission cost of } \\ & \text { wired link }\end{array}$

According to RFC3775[1] and RFC4140 [2] in MIPv6 there is only home registration but in HMIPv6 there are to registrations: regional registration and home registration. Hence, $\mathrm{T}_{\mathrm{RM}}, \mathrm{T}_{\text {intra }}$ and $\mathrm{T}_{\text {inter }}$ can be calculated as:

$\mathrm{T}_{\mathrm{RM}}=2 \mathrm{~T}_{\mathrm{AM}}+2 \mathrm{~T}_{\mathrm{HA}}+\mathrm{T}_{\mathrm{H}}$

$\mathrm{T}_{\text {intra }}=2 \mathrm{~T}_{\mathrm{AM}}+2 \mathrm{~T}_{\mathrm{MA}}+\mathrm{T}_{\mathrm{M}}$

$\mathrm{T}_{\text {inter }}=4 \mathrm{~T}_{\mathrm{AM}}+2 \mathrm{~T}_{\mathrm{MA}}+2 \mathrm{~T}_{\mathrm{HA}}+\mathrm{T}_{\mathrm{H}}+\mathrm{T}_{\mathrm{M}}$

Let the MN needs $\mathrm{m}^{\text {th }}$ handover to move out of a region $(\mathrm{m} \geq 1)$.Then, in new region the $\mathrm{MN}$ will enter at its $\mathrm{m}^{\text {th }}$ handover. So the total average delay $\left(\mathrm{T}_{\mathrm{IT}}\right)$ that an $\mathrm{MN}$ spends for $\mathrm{m}$ handovers in HMIPv6 and MIPv6 is [3]

$\mathrm{T}_{\mathrm{IT}}=(\mathrm{m}-1) \mathrm{T}_{\text {intra }}+\mathrm{T}_{\text {inter }}$

$\mathrm{T}_{\mathrm{AT}}=\mathrm{m} \cdot \mathrm{T}_{\mathrm{RM}}$

Using definition 3.1 and equations (4) \& (5), $T_{R}$, can be calculated as

$$
T_{R}=\frac{\mu\left(2 \theta+2 m l_{M A}-2 l_{H A}(m-1)+m\left(T_{M}-T_{H}\right)+T_{H}\right)}{m T}
$$

Where $\mu$ is unit distance signaling transmission cost of of wired link. We also suppose the average signaling delivering delay of wireless link be $\theta$. $\mu$, where $\theta>1$. From formulae (6) we can say that if the nearer the distance between $\mathrm{MN}$ and MAP and the farther the distance between HA and MN, then HMIPv6 gives higher average registration revenue. i.e. Only when, $\mathrm{T}_{\mathrm{R}}<0$, HMIPv6 obtains the average registration revenue. Two theorems can be deduced.

Theorem 1: HMIPv6 outperforms MIPv6 in terms of registration revenue when an $\mathrm{MN}$ roams within a region (intra-region) and the average registration revenue is $\left|2 \mu .\left(l_{\mathrm{MA}}-1_{\mathrm{HA}}\right) / \mathrm{T}\right|$. In micro-mobility $\mathrm{T}_{\mathrm{R}}$ can be calculated as [4]

$T_{R}=\frac{2 \mu\left(l_{M A}-l_{H A}\right)+T_{M}-T_{H}}{T}$

Theorem 2: $T_{R}$ lies on the regional size, $K$, when the $M N$ roams across different regions (inter-region). In this $T_{R}$ can be calculated as on certain conditions as [4]:

$$
\begin{gathered}
T_{R}=\frac{\left(2 \mu \cdot \theta+T_{H}\right) \cdot(2 N-2 K-1)+2 \mu \cdot l_{H A} \cdot(1-2 K)}{(2 N-2) \cdot T} \\
+\frac{4 \mu \cdot(N-1) \cdot l_{M A}+2(N-1) \cdot\left(T_{M}-T_{H}\right)}{(2 N-2) \cdot T}
\end{gathered}
$$

\subsection{Relative Packet Delivery Cost [3]}

\section{Definition 3.2 (Relative Packet Delivery Cost)}

Relative packet delivery cost $\left(D_{P}\right)[3]$ is defined as the average time wasted by using HMIPv6 instead of MIPv6 to forward packets.

Assume that the average packet delivery delay of wired link is proportional to the number of hops that the packets travel with the proportionality constant $\eta$. Then Equation becomes [IMS]

$\mathrm{D}_{\mathrm{P}}=\alpha \cdot\left(\mathrm{AwK}+\mathrm{BlgK}+\delta+\eta \cdot\left(\mathrm{l}_{\mathrm{HM}}+\mathrm{l}_{\mathrm{MA}}-\mathrm{l}_{\mathrm{HA}}\right)\right)$

Where $\alpha$ is average packet arrivel time, $\delta$ is the average delay of encapsulating a packet in MAP, A \& B are coefficients, w.k is the average no. ARs in a region with assuming that an AR can serve w MNs on average and $\mathrm{lg}$ is the logarithmic function.

Equation (9) leads to the conclusion that average packet delivery cost is positive on certain condition [4]. When $D_{P}>0$, it means average packet delivery delay of HMIPv6 is longer than that of MIPv6.

\subsection{Relative Cost [3]}

\section{Definition 3.2 (Total Cost Function)}

Total cost function denoted as $C_{T}$ gives the overall performance of HMIPv6 against MIPv6 in terms of registration and packet delivery cost [3].

$$
\mathrm{C}_{\mathrm{T}}=\mathrm{n}_{1} \cdot \mathrm{T}_{\mathrm{R}}+\mathrm{n}_{2} \cdot \mathrm{D}_{\mathrm{P}}
$$

Where $\mathrm{n}_{1}>0$ and $\mathrm{n}_{2}>0$ are the coefficients.

As per the eq. (10), when $C_{T}>0$, MIPv6 will be more applicable than HMIPv6 otherwise HMIPv6 is adopted.

\section{PERFORMANCE EVALUATION OF MIPV6 AND HMIPV6}

In this section we employ an algorithm to evaluate the performance of MIPv6 and HMIPv6 against some key parameters using an analytical model. The algorithm of the impact of parameters on the applicability of MIPv6 and HMIPv6 has been described in this section.

\subsection{Algorithm for the performance of MIPv6 \& HMIPv6}

1. Enter the $x$ different values of Regional Size $(\mathrm{K})$ and a fixed number of Access Routers (N).

2. Enter the fixed values of $\mathrm{T}, \alpha$ and $\mathrm{l}_{\mathrm{MA}}$

3. for ( $\mathrm{i}=1$ to $x$ )

$\boldsymbol{I F}$ (Regional size [i] $\geq \mathrm{N}$ (no. of different access routers)

Compute $\mathrm{T}_{\mathrm{R}}$ according to eq. (7).

Compute $\mathrm{D}_{\mathrm{P}}$ according to eq. (9).

Compute $\mathrm{C}_{\mathrm{T}}$ according to eq. (10).

Plot a graph between $C_{T}$ and Regional size 
ELSE

Compute $\mathrm{T}_{\mathrm{R}}$ according to eq. (8).

Compute $\mathrm{D}_{\mathrm{P}}$ according to eq. (9).

Compute $\mathrm{C}_{\mathrm{T}}$ according to eq. (10).

Plot a graph between $\mathrm{C}_{\mathrm{T}}$ and Regional size

4. Change $\mathrm{T}$ to see the impact of $\mathrm{T}$ on $\mathrm{C}_{\mathrm{T}}$ for a fixed value of regional size and go to step 3 .

5. Change $\alpha$ to see the impact of $\alpha$ on $\mathrm{C}_{\mathrm{T}}$ for a fixed value of regional size and go to step 3 .

6. Change $l_{\mathrm{MA}}$ to see the impact of $\mathrm{l}_{\mathrm{MA}}$ on $\mathrm{C}_{\mathrm{T}}$ for a fixed value of regional size and go to step 3 .

7. Exit.

\section{NUMERICAL RESULTS AND DISCUSSION}

In this section, we demonstrate the performance of MIPv6 and HMIPv6 against some key parameters. We employ numerical analysis to show the results. The parameters used in the simulation process are taken from the various existing literature. The estimating value of $\alpha$ can be found in $[7,8]$ while $\mathrm{T}$ can be computed by the method introduced in [6]. In addition the value of $\mathrm{w}$ and $\mathrm{N}$ are from [10]. The estimating value of $l_{\mathrm{HM}}$ and $l_{\mathrm{MA}}$ are taken from [9].

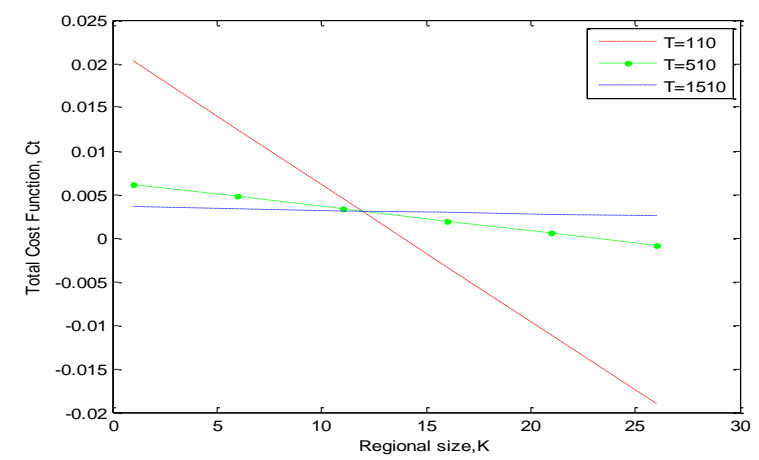

Fig 1: Impact of $T$ on $C_{T}$

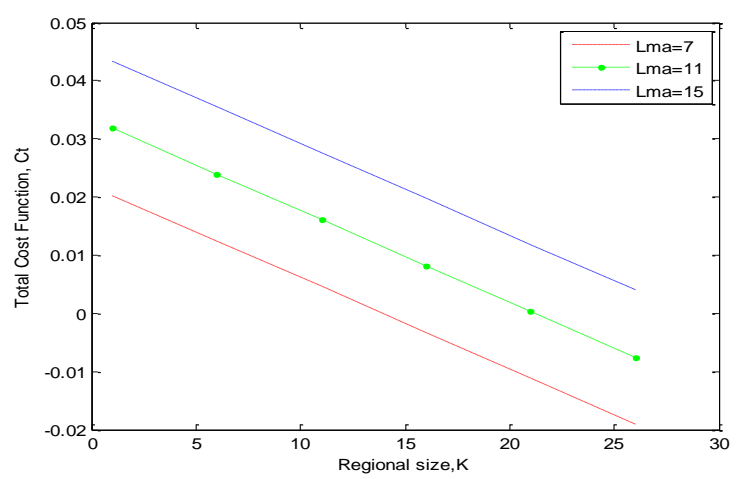

Fig 2: Impact of $\alpha$ on $\mathbf{C}_{\mathbf{T}}$

In figure 1 impact of $\mathrm{T}$ on $\mathrm{C}_{\mathrm{T}}$ has been shown. In this case the values of $1_{\mathrm{MA}}=7$ and $\alpha=.06$. In this figure we see that for $\mathrm{K} \leq$ $13, \mathrm{C}_{\mathrm{T}}$ is greater than 0 and increases with the decreasing of $\mathrm{T}$. In figure 2 , the impact of $\alpha$ on $\mathrm{C}_{\mathrm{T}}$ has been shown. In this case the values of $1_{\mathrm{MA}}=7$ and $T=110$. Since as $\alpha$ increases the average packet delivery cost is increased which leads to increase in $\mathrm{C}_{\mathrm{T}}$.

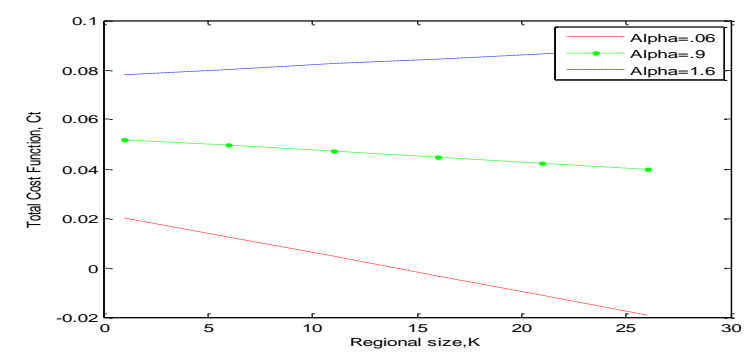

Fig 3: Impact of $\mathrm{l}_{\mathrm{MA}}$ on $\mathrm{C}_{\mathrm{T}}$

Figure 3 shows the impact of the distance $\left(1_{\mathrm{MA}}\right)$ between AR and MAP on $\mathrm{C}_{\mathrm{T}}$. In this case the values of $\mathrm{T}=110$ and $\alpha=.06$ is taken. In this figure we see that as $1_{\mathrm{MA}}$ is increased, the $\mathrm{C}_{\mathrm{T}}$ is also increased. This is due to the fact that both average packet delivery delay and the average registration delay is increased in HMIPv6 when the distance $1_{\mathrm{MA}}$ increases, leading in the increase in $\mathrm{C}_{\mathrm{T}}$.

\section{CONCLUSION}

In this paper we evaluated a performance of MIPv6 and HMIPv6 in the form of impact of certain parameters on the total cost function. It is also concluded that although HMIPv6 is a modification of MIPv6, but it cannot outperforms MIPv6 always. Finally the performance of MIPv6 and HMIPv6 has been simulated in this paper.

\section{REFERENCES}

[1] D. Johnson, C. Perkins, and J. Arkko, "Mobility Support in IPv6," RFC3775, June 2004.

[2] H. Soliman, C. Castelluccia, K. El Malki, and L. Bellier, "Hierarchical Mobile IPv6 Mobility Management (HMIPv6)," RFC4140, 2005.

[3] Shengling Wang, Yong Cui, Sajal K. Das, Wei Li, and Jianping $\mathrm{Wu}$, "Mobility in IPv6: Whether and How to Hierarchize the Network?" 1045-9219/11/\$26.00 , 2011 IEEE

[4] Shengling Wang, Yong Cui, Sajal K.Das "Intelligent Mobility support for IPv6". 978-19/08/\$25.00 @2008 IEEE

[5] H. Tzeng and T. Przygienda. "On Fast Address-Lookup Algorithms".IEEE J. Selected Areas in Communications, vol. 17, no. 6, pp. 1067-1082, 1999.

[6] Y. Chen and M. Huang. "A Novel MAP Selection Scheme by Using Abstraction Node in Hierarchical MIPv6". Proc. IEEE International Conference on Communications, 2006. pp:5408-5413

[7] H. Xie, S. Tabbane, and D.J. Goodman. "Dynamic Location Area Management and Performance Analysis". Proc. $43^{\text {rd }}$ IEEE Vehicular Technology Conference, 1993. pp:536-539

[8] M. Yabusaki. "Mobility/Traffic Adaptive Location Management". Proc. IEEE 56th Vehicular Technology Conference, Vancouver, 2002. pp:1011-1015

[9] W.R. Stevens, TCP/IP Illustrated, Volume 1: The Protocols. Addison Wesley Longman, Inc., 1994.

[10] J Xie. and I.F.Akyildiz. "A Novel Distributed Dynamic Location Management Scheme for Minimizing Signaling Costs in Mobile IP". IEEE Transactions on Mobile Computing, vol. 1, no. 3, pp.163-175,2002. 